\title{
DINAMICA DE LA CUERDA RELATIVISTA CERCA DE UN AGUJERO NEGRO
}

\author{
Fulgencio Villegas Silva ${ }^{a}$ \\ ${ }^{a}$ Departamento de Física Atómica y Nuclear - Facultad de Ciencias Físicas \\ Universidad Nacional Mayor de San Marcos. Apartado Postal 14-0149, Lima 14, Perú.
}

\begin{abstract}
Resumen
Se estudia la dinámica de una cuerda cerca de un agujero negro de Kaluza-Klein. Las soluciones a las ecuaciones geodésicas se obtienen usando la velocidad de la luz en la hoja de mundo como un parámetro de expansión. Para una cuerda que entra en un agujero negro magnéticamente cargado, se demuestra que la dimensión compacta decrece con la coordenada $\tau$ de la hoja de mundo.
\end{abstract}

Palabras claves: Teoría de Kaluza-Klein, agujero negro, dinámica de cuerdas, compactificación.

\begin{abstract}
The dynamics of a string near a Kaluza-Klein black hole are studied. Solutions to the geodesic equations are obtained using the world sheet velocity of light as an expansion parameter. For a string falling into a magnetically charged black hole, it is shown that the compact dimension decreases with the world-sheet coordinate $\tau$.
\end{abstract}

Keywords: Kaluza-klein theory, black-hole, string dynamics, compactification.

\section{Introducción}

La teoría de cuerdas [1] ha hecho recientemente contribuciones importantes al estudio de los agujeros negros y el background cosmológico. Desde que una cuerda posee grados internos de libertad, la dinámica es fundamentalmente diferente del caso de una partícula. Una cuerda describe con su movimiento una hoja de mundo contrario a lo que una partícula describe con su movimiento una línea de mundo, por consiguiente las ecuaciones de movimiento de una cuerda están parametrizadas por dos variables $\sigma$ y $\tau$. La dinámica de la cuerda está gobernada por la siguiente acción

$$
S=-T_{0} \int d \tau d \sigma \sqrt{-\operatorname{det} g_{a b}}
$$

donde $g_{a b}=G_{\mu v}(X) \partial_{a} X^{\mu} \partial_{b} X^{v}$ es la métrica bidimensional de la hoja de mundo. Se puede usar una invariancia en la representación de la hoja de mundo considerando el gauge conforme $g_{a b}=\rho(\sigma, \tau) \eta_{\mu v}$ donde $\eta_{\mu v}$ es la métrica bidimensional de Minkowski.. Las ecuaciones clásicas de movimiento en este gauge están dadas por

$\partial_{\tau}^{2} X^{\mu}-c^{2} \partial_{\sigma}^{2} X^{\mu}+\Gamma_{v \rho}^{\mu}\left[\partial_{\tau} X^{v} \partial_{\tau} X^{\rho}-c^{2} \partial_{\sigma} X^{v} \partial_{\sigma} X^{\rho}\right]=0$.

con las siguientes condiciones

Email: fvillegass@unmsm.edu.pe 


$$
\begin{aligned}
\partial_{\tau} X^{\mu} \partial_{\sigma} X^{v} G_{\mu \nu} & =0 \\
{\left[\partial_{\tau} X^{\mu} \partial_{\tau} X^{\nu}+c^{2} \partial_{\sigma} X^{\mu} \partial_{\sigma} X^{\nu}\right] G_{\mu \nu} } & =0
\end{aligned}
$$

dónde $c$ es la velocidad de la luz en la hoja de mundo. El sistema de ecuaciones es no lineal, Para resolver estas ecuaciones usamos varios ansatze que las simplifican. Seguimos las indicaciones sugeridas en [3], donde las coordenadas de la cuerda son expandidas usando la velocidad de la luz en la hoja de mundo como un parámetro de expansión. En el límite de $c$ pequeño, la derivada con respecto a $\tau$ domina sobre la derivada respecto a $\sigma$. Como es opuesto al límite de valores grandes de $c$, esta da un espectro dinámico. Si $c<<1$, la coordenada de expansión es conveniente para describir la cuerda en un background gravitacional fuerte [4,5]. Este límite corresponde al límite de tensión nula de la cuerda, donde la velocidad de la luz en la hoja de mundo es proporcional a la tensión de la cuerda.

\section{Agujero negro de Kaluza-Klein y dinámica de la cuerda}

Considerando el caso dónde la velocidad de la luz en la hoja de mundo es pequeña, las coordenadas de la cuerda son expresadas como

$$
X^{\mu}(\sigma, \tau)=A^{\mu}(\sigma, \tau)+c^{2} B^{\mu}(\sigma, \tau)+c^{4} C^{\mu}(\sigma, \tau)+\ldots
$$

y resolviendo la ecuación de movimiento para el orden cero $A^{\mu}(\sigma, \tau)$. Estas ecuaciones describen la energía de la cuerda correspondiente al límite de tensión nula. La métrica para estudiar la propagación de la cuerda [6] es

$d s^{2}=-e^{4 k \frac{\varphi}{\sqrt{3}}}\left(d x_{5}+A_{\alpha} d x^{\alpha}\right)^{2}+e^{-2 k \frac{\varphi}{\sqrt{3}}} g_{\alpha \beta} d x^{\alpha} d x^{\beta}$,

donde $k^{2}=4 \pi G, x_{5}$ es la dimensión extra y debe identificarse con el modulo $2 \pi R_{0}$. Donde $g_{\alpha \beta}$ es el espacio-tiempo tetra-dimensional. Las soluciones independientes del tiempo del agujero negro de Kaluza-Klein son esféricamente simétricas y están caracterizadas por su masa M, Carga eléctrica total Q y carga magnética $\mathrm{P}$ [6]. Por simplicidad se considera agujeros negros eléctricamente neutros. Las ecuaciones de movimiento en el caso puramente magnético pueden reducirse a cuadraturas si restringimos el movimiento de la cuerda en el plano ecuatorial, esto es $\theta=\pi / 2$. Las integrales se evaluarán numéricamente para obtener las coordenadas de la cuerda en términos de la coordenada $\tau$ de la hoja de mundo. Se analiza en la región donde $\mathrm{r}>>\Sigma, \Sigma$ es la carga escalar total y $r>M$. Esto es justamente la región fuera del horizonte, como una función de $\tau$. La cantidad físicamente interesante para estudiar cómo la dimensión compacta extra se despliega es el radio de Kaluza-Klein el cual es definido como

$$
R(r)=R_{0} e^{2 k \varphi / \sqrt{3}}
$$

$R(r)$ es una cantidad dinámica y depende explícitamente de $\tau$ como $R(\tau)=R(r(\tau))$.El efecto del campo magnético es encogerse la dimensión extra [6] es decir, como la cuerda se acerca el agujero negro, el valor del radio de Kaluza-Klein, se pone más pequeño que su valor asintótico; como se ilustra en la figura 1.

\section{Conclusiones}

Se ha estudiado la propagación de una cuerda nula hacia un agujero negro de Kaluza-Klein magnéticamente cargado. Se ha analizado el comportamiento de la quinta dimensión extra a medida que la cuerda se aproxima al horizonte de eventos del agujero negro. Las soluciones que se han obtenido son válidas en la región fuera del horizonte pero no asintóticamente lejos del Horizonte. Cabe indicar que las soluciones obtenidas, en el límite $\Sigma \rightarrow 0$ resultan ser las mismas calculadas en [5]. Las consideraciones anteriores muestran claramente que incluso en el régimen clásico se puede explorar el comportamiento de las dimensiones extra.

\section{Referencias}

[1] M. B. Green, J. H. Schwarz and E. Witten, Superstring theory, Cambridge Univ. Press, 1978. 
[2] Boris Pioline, Lectures on black holes, topological strings an quantum attractors, hep-th/ 0607227.

H. J. De Vega and N. Sanchez, hep-th/ 9512074.

[3] Renata Kallosh, Andrei Linde, Strings, black holes, and quantum information, hep-th/0602061.

H. J. De Vega and A. Nicolaidis, Phys. Lett. B295, 241 (1992).

[4] H. J. De Vega, I Giannakis and D. L. Wiltshire, mod. Phys. Lett. A10, 2479 (1995).
[5] C. O. Lousto and N. Sánchez, Phys. Rev. D54, 6399 (1996).

[6] Lorenzo Carnalba, Miguel S. Costa, João Peredones, Pedro Vieira, From fundamental strings tos mall black holes. Hep-th/ 0607083.

Thomas Mohaupt, Strings, higher curvatura consctions, and black-holes. Hep-th/ 0512048.

G.W. Gibbons and D. L. Wiltshire, Ann. Phys. 167, 201 (1987); 176, 393(E) (1987).

[7] N. Itzhaki, Nucl. Phys. B580, 700 (1997).

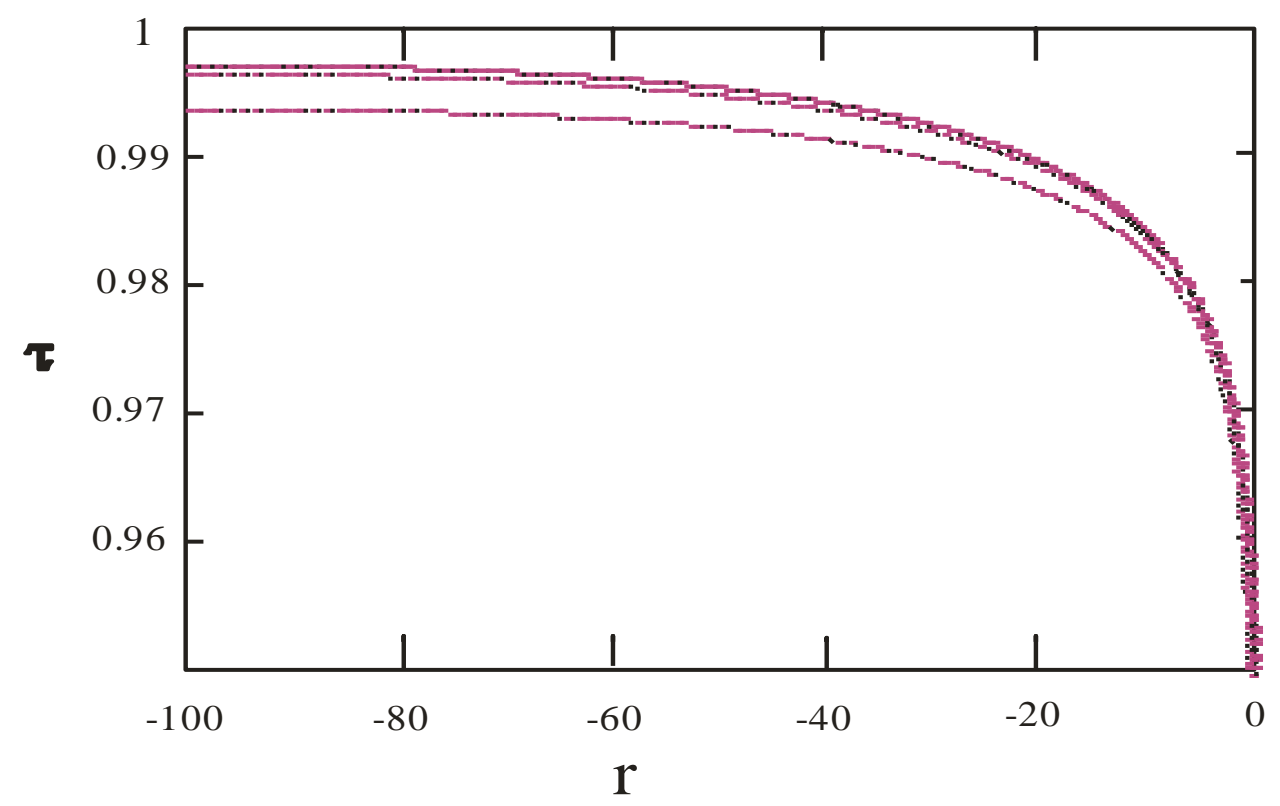

Figura 1. Radio de Kaluza-Klein en función de $\tau$ 